\title{
STRONG LAWS OF LARGE NUMBERS FOR ARRAYS OF ROW-WISE EXCHANGEABLE RANDOM ELEMENTS
}

\author{
ROBERT LEE TAYLOR \\ Department of Statistics and Computer Science \\ University of Georgia \\ Athens, GA 30602
}

RONALD FRANK PATTERSON

Department of Mathematics

Georgia State University

At lanta, GA 30303

(Received October 1, 1984)

ABSTRACT. Let $\left\{x_{n k}: 1 \leq k \leq n, n \geq 1\right\}$ be a triangular array of row-wise exchangeable random elements in a separable Banach space. The almost sure convergence of $n^{-1 / l_{\sum}}{ }_{k=1}^{n}$ $\mathrm{x}_{\mathrm{nk}}, 1 \leq \mathrm{p}<2$, is obtained under varying moment and distribution conditions on the random elements. In particular, strong laws of large numbers follow for triangular arrays of random elements in (Rademacher) type p separable Banach spaces. Consistency of the kernel density estimates can be obtained in this setting.

KEY WORDS AND PHRASES. Exchangeability, Random Elements, Laws of Large Numbers, Almost sure Convergence, Martingales, Rademacher type p, and Kernel Density Estimates.

1980 MATHEMATICS SUBJECT CLASSIFICATION CODES. Primary 60B12, Secondary $60 B 11$.

1. INTRODUCTION AND PRELIMINARIES.

Blum et a1. [1] obtained central limit theorems for arrays of exchangeable random variables using a version of de Finetti's theorem which implied that an infinite sequence of exchangeable random variables is a mixture of sequences of independent, identically distributed random variables. Taylor [2] used similar techniques in obtaining weak and strong convergence results for arrays of random elements which are row-wise exchangeable. Using martingale methods, Weber [3] developed central limit results for triangular arrays of random variables which were row-wise exchangcable. His methods did not require infinite exchangeability or the de Finetti representation. In this paper, almost sure convergence is obtained for $\frac{1}{n} \sum_{k=1}^{n} x_{n k}$ and $n^{-1 / p} \sum_{k=1}^{n} x_{n k}$ in separable Banach spaces using martingale methods. These results 
are for triangular arrays, and hence only finite exchangeability in each row is required. By assuming convergence in mean for each column, the hypothesis of the previously cited limit theorems are substantially relaxed.

Let $E$ denote a real separáble Banach space with norm \|\| . Let $(\Omega, A, P$ ) denote a probability space. A random element $X$ in $E$ is a function from $\Omega$ into $E$ which is A-measurable with respect to the Borel subsets of $E, B(E)$. The pth absolute moment of a random element $X$ is $E|| X \|^{p}$ where $E$ is the expected value of the (real-valued) random variable $\|x\|^{P}$. The expected value of $x$ is defined to be the Bochner integral (when $\mathrm{E}|| \mathrm{X}||<\infty$ ) and is denoted by EX. The concepts of independence and identical distributions (i.i.d.) have direct extensions to $E$. The random elements $\left\{x_{1}, \ldots x_{n}\right\}$ are said to be exchangeable if the joint probability law of $\left(x_{1}, \ldots, x_{n}\right)$ is permutation invariant, that is, for each permutation $\pi$ of $\{1, \ldots, n\}$

$$
P\left[x_{1} \in B_{1}, \ldots, x_{n} \in B_{n}\right]=P\left[X_{\pi 1} \varepsilon B_{1}, \ldots, x_{\pi n} \in B_{n}\right]
$$

for each $B_{1}, \ldots, B_{n} \in B(R)$. Clearly i.i.d. random elements are exchangeable but not conversely. Moreover, letting $B_{k+1}=\ldots=B_{n}=R$ for $1 \leq k \leq n$ in (1.1) shows that all joint probability laws are the same and that exchangeable random elements are identically distributed. Finally, a subset $B$ of $E$ whose boundary $\partial B$ satisfies $P(\partial B)=0$ is called a P-continuity set.

\section{STRONG LAWS OF LARGE NUMBERS FOR TRIANGULAR ARRAYS.}

The main result of this section is a strong law of large numbers. Moment conditions and a measure of nonorthogonality condition will be assumed on the distributions of the random elements. Throughout this section $\left\{x_{n k}: 1 \leq k \leq n, n \geq 1\right\}$ will denote an array of random elements in a separable Banach space $E$ which are row-wise exchangeable.

First, two preliminary results will be presented for 1 ater use in Sections 2 and 3. The first result shows that the infinite sequence, formed by the convergence in rth mean of each column of the triangular array, is exchangeable when each row consists of exchangeable random elements. This allows the application of a version of de Finetti's theorem to the limit sequence.

LEMMA 1. Let $\left\{\mathrm{x}_{\mathrm{nk}}: 1 \leq \mathrm{k} \leq \mathrm{n}, \mathrm{n} \geq 1\right\}$ be an array of random elements which are row-wise exchangeable. If the random elements converge in the $r$ th mean to $x_{\infty k}(r>0)$ for each $k$, then the sequence $\left\{x_{\infty k}: k \geq 1\right\}$ is exchangeable. 
PROOF: Consider the set $\left\{x_{n 1}, \ldots, x_{n k}\right\} \subset\left\{x_{n 1}, \ldots, x_{n n}\right\}$ and the vector $\left(x_{n l}, \ldots, x_{n k}\right)$. If $x_{n i} \stackrel{r}{\rightarrow} x_{\infty i}, 1 \leq i \leq k$, then $\left(x_{n l}, \ldots, x_{n k}\right) \stackrel{r}{\rightarrow}\left(x_{\infty 1}, \ldots, x_{\infty k}\right)$. Hence, $\left(x_{n 1}, \ldots, x_{n k}\right) \stackrel{D}{\rightarrow}\left(x_{\infty l}, \ldots, x_{\omega \times k}\right)$. For each $P_{x_{i x}}$ - continuity set $A_{i}$ of 1 , $1 \leq i \leq k,\left([4]\right.$ pp. 26-27), $P\left[X_{n 1} \in A_{1}, \ldots, X_{n k} \varepsilon A_{k}\right] \rightarrow P\left[X_{\infty} \mid \varepsilon A_{1}, \ldots, X_{\infty} \varepsilon A_{k}\right]$. By exchangeability, $P\left[X_{n 1} \varepsilon A_{1}, \ldots, X_{n k} \varepsilon A_{k}\right]=P\left[X_{n \pi l} \varepsilon A_{1}, \ldots, X_{n \pi k} \varepsilon A_{k}\right]$ for each permutation $\pi$ of $(1, \ldots, k)$. Hence, $P\left[X_{n \pi l} \varepsilon A_{1}, \ldots, X_{n \pi k} \varepsilon A_{k}\right] \rightarrow$ $\mathrm{P}\left[\mathrm{X}_{\infty \pi \mathrm{l}} \in \mathrm{A}_{1}, \ldots, \mathrm{X}_{\infty \pi \mathrm{k}} \in \mathrm{A}_{\mathrm{k}}\right]$ as $\mathrm{n} \rightarrow \infty$ for all $\mathrm{A}_{1}, \ldots, \mathrm{A}_{\mathrm{k}}$ which are $\mathrm{P}_{\mathrm{X}_{\infty} \mathrm{l}^{- \text {continuity }}}$ sets. Since the limits are unique, and the $\mathrm{P}_{\mathrm{X}_{\infty} 1}$-continuity sets form a determining lass, it follows that

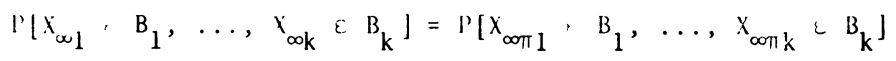

for all $\left(B_{1}, \ldots, B_{k}\right) \in B\left(E^{k}\right)$. Thus, $\left(X_{\infty 1}, \ldots, X_{\infty k}\right)$ and $\left(X_{\infty \pi 1}, \ldots, X_{\infty \pi k}\right)$ have identical joint distributions. Hence, the sequence $\left\{x_{\text {ook }}: k \geq 1\right\}$ is exchangeable. ///

REMARK. Note that the convergence of the joint distributions is sufficient in the proof of Lemma 1. Unfortunately, this is not implied by convergence in distribution in each column.

For arrays where each row is an infinite sequence of exchangeable random elements, Olshen [5] showed that de Finetti's theorem implied that for each $n$

$$
P\left(B_{n}\right)=\int_{F} P_{\nu}\left(B_{n}\right) d \mu_{n}\left(P_{\nu}\right)
$$

where $F$ denotes the collection of probabilities on the Borel subsets of $E$ and $P_{V}\left(B_{n}\right)$ is the probability of $B_{n}=\left[g\left(X_{n l}, \ldots, x_{n m}\right) \varepsilon B_{n}\right]$ (where $g: E^{m} \rightarrow E$ is a Borel function) computed under the assumption that $\left\{x_{n k}: k \geq 1\right\}$ are independent, identically distributed random elements and $\mu_{n}$ is the mixing measure defined on $B(F)$. The next result shows that if $\left\{x_{n k}: 1 \leq k \leq n, n \geq 1\right\}$ are row-wise exchangeable random elements which converge in the second mean for each $k$ and $E\left[f\left(X_{n 1}\right) f\left(X_{n 2}\right)\right] \rightarrow 0$ as $n \rightarrow \infty$ then $E\left[f\left(X_{\infty 1}\right) f\left(X_{\infty 2}\right)\right]=0$ and $E_{v}\left(X_{\infty 1}\right)=0$ where $E_{\nu}$ is the expectation with respect to $\mathrm{P}_{v^{\prime}}$ Moreover, it also follows that $\mathrm{Ex}_{\infty 1}=0$.

LEMMA 2. Let $\left\{x_{n k}: 1 \leq k \leq n, n \geq 1\right\}$ be an array of row-wise exchangeable random elements in a separable Banach space such that $\left\{x_{n k}\right\}$ converges in the second mean to $x_{\infty k}$ for each $k$. If for each $f \varepsilon E^{*}$

$$
\rho_{n}(f)=L\left[f\left(x_{n 1}\right) f\left(x_{n 2}\right)\right] \rightarrow 0 \text { as } n \rightarrow \infty \text {, }
$$

then

$$
E\left[f\left(x_{\infty 1}\right) f\left(x_{\infty 2}\right)\right]=0
$$


(ii) $E_{V}\left(X_{\infty 1}\right)=0 \quad$ for $P_{v}$ with $\mu_{\infty}$-probability one, and

(iii) $E\left(X_{\infty 1}\right)=0$.

PROOF. Since $x_{n k} \stackrel{2}{\rightarrow} x_{\infty k}$ for each $k$, it is clear that

$$
\sup _{n} E|| X_{n k}||^{2}<\infty \text { and } E \| x_{\infty k}||^{2}<\infty
$$

for each $k$. Using (2.2) and convergence in the 2nd mean, for each $f \in E^{*}$

$$
\begin{aligned}
& \left|E\left[f\left(x_{n 1}\right) f\left(x_{n 2}\right)\right]-E\left[f\left(x_{\infty 1}\right) f\left(x_{\infty 2}\right)\right]\right| \\
& \leq \mid E\left[f\left(x_{n 1}\right) f\left(x_{n 2}\right)-E\left[f\left(x_{\infty 1}\right) f\left(x_{n 2}\right)\right] \mid\right. \\
& +\left|E\left[f\left(x_{\infty 1}\right) f\left(x_{n 2}\right)\right]-E\left[f\left(x_{\infty 1}\right) f\left(x_{\infty 2}\right)\right]\right| \\
& \leq E\left(\left|f\left(x_{n 1}\right)-f\left(x_{\infty 1}\right)\right|\left|f\left(x_{n 2}\right)\right|\right) \\
& +E\left(\left|f\left(x_{n 2}\right)-f\left(x_{\infty 2}\right)\right|\left|f\left(x_{\infty 1}\right)\right|\right) \\
& \leq\|f\|^{2}\left[E\left(\left\|x_{n 1}-x_{\infty 1}\right\|\left\|x_{n 2}\right\|\right)+E\left(\left\|x_{n 2}-x_{\infty 2}\right\|\left\|x_{\infty 1}\right\|\right)\right] \\
& \leq\|f\|^{2}\left[\left(E|| x_{n 1}-x_{\infty 1} \|^{2}\right)^{1 / 2}\left(E|| x_{n 2} \|^{2}\right)^{1 / 2}\right. \\
& \left.+\left(E|| x_{n 2}-x_{\infty 2} \|^{2}\right)^{1 / 2}\left(E|| x_{\infty 1} \|^{2}\right)^{1 / 2}\right]
\end{aligned}
$$

which goes to 0 as $n \rightarrow \infty$ by hypothesis (i). Thus,

$$
E\left[f\left(x_{\infty 1}\right) f\left(x_{\infty 2}\right)\right]=0
$$

Since convergence in the second mean implies convergence in mean, it follows from Lemma 1 that the sequence $\left\{x_{\infty k}: k \geq 1\right\}$ is exchangeable. Also, it follows from (2.1) that

$$
\begin{aligned}
0 & =E\left[f\left(X_{\infty 1}\right) f\left(X_{\infty 2}\right)\right] \\
& =\int_{F} E_{\nu}\left[f\left(X_{\infty 1}\right) f\left(X_{\infty 2}\right)\right] d \mu_{\infty}\left(P_{\nu}\right) \\
& =\int_{F}\left[E_{\nu} f\left(X_{\infty 1}\right)\right]^{2} d \mu_{\infty}\left(P_{\nu}\right)
\end{aligned}
$$

which implies that $E_{\nu} f\left(X_{\infty l}\right)=0 \mu_{\infty}$ a.s. for each $f \varepsilon E^{\star}$. Since the Bochner integral implies the Pettis integral in a separable space, $f\left(E_{v}\left(X_{\infty 1}\right)\right)=E_{\nu}\left(f\left(X_{\infty 1}\right)\right)=0 \mu_{\infty}$ a.s. for each $f \in E^{*}$ which implies that $E_{V}\left(X_{\infty 1}\right)=0 \mu_{\infty}$ a.s. by the Hahn-Banach theorem and the separability of E. Also,

$$
E X_{\infty 1}=\int_{F} E_{v}\left(X_{\infty 1}\right) d \mu_{\infty}\left(P_{v}\right)=0
$$

REMARK. Note from (2.3) in the proof of Lemma 2 that for uniformly bounded random elements, convergence in the mean suffices. It is also interesting to note 
that the conclusions of Lemma 2 do not necessarily imply that $\left\{x_{\infty k}: k \geq 1\right\}$ is a sequence of i.i.d. random elements.

The final result of this section is a strong law of large numbers for triangular arrays of random elements which are row-wise exchangeable. Define

$$
\begin{aligned}
& \mathrm{U}_{\mathrm{nn}}=\sigma\left\{\sum_{\mathrm{k}=1}^{\mathrm{n}} \mathrm{x}_{\mathrm{nk}}, \sum_{\mathrm{k}=1}^{\mathrm{n}+1} \mathrm{x}_{(\mathrm{n}+1) \mathrm{k}}, \ldots\right\} \text { and } \\
& \mathrm{U}_{\infty \mathrm{n}}=\sigma\left\{\sigma\left\{\sum_{\mathrm{k}=1}^{\mathrm{n}} \mathrm{x}_{\infty \mathrm{k}}, \sum_{\mathrm{k}=1}^{\mathrm{n}+1} \mathrm{x}_{\infty \mathrm{k}}, \ldots\right\} \cup \mathrm{U}_{\mathrm{nn}}\right\}
\end{aligned}
$$

It can be casily shown that if ||$x_{n 1}-x_{\infty 1}\|\geq\| x_{(n+1), 1}-x_{\infty 1} \|$ for each $n$ and $\mathrm{x}_{\mathrm{nk}} \stackrel{2}{\rightarrow} \mathrm{x}_{\infty \mathrm{k}}$ for each $\mathrm{k}$, then

$$
E\left(|| x_{n k}-x_{\infty k} \| u_{\infty n}\right) \rightarrow 0 \text { a.s. }
$$

Using (2.4), a modification of Kingman's [6] and Weber's [3] results show that

$$
\begin{aligned}
& \frac{1}{n} \sum_{k=1}^{n} X_{n k}=E\left(X_{n 1} \mid U_{n n}\right)=E\left(X_{n 1} \mid U_{\infty n}\right)=\text { a.s. and } \\
& \frac{1}{n} \sum_{k=1}^{n} X_{\infty k}=E\left(X_{\infty 1} \mid U_{\infty n}\right) \text { a.s. }
\end{aligned}
$$

THEOREM 1. Let $\left\{\mathrm{x}_{\mathrm{nk}}: 1 \leq \mathrm{k} \leq \mathrm{n}, \mathrm{n} \geq 1\right\}$ be an array of random elements in a separable Banach space. Let $\left\{x_{n k}\right\}$ be row-wise exchangeable for cach $n$ and lct $U_{n n}$ and $U_{\infty n}$ be the $\sigma$-fields defined in (2.4). Let $\left\{x_{n k}\right\}$ converge in the second mean for each $k$ and $\left\|x_{n 1}-x_{\infty 1}\right\| \geq\left\|x_{(n+1), 1}-x_{\infty 1}\right\|$ for each $n$. If

$$
\rho_{n}(f)=E\left[f\left(X_{n 1}\right) f\left(X_{n 2}\right)\right] \rightarrow 0 \text { as } n \rightarrow \infty \text { for each } f \varepsilon E^{*} \text {, }
$$

then

$$
\left\|\frac{1}{n} \sum_{k=1}^{n} x_{n k}\right\| \rightarrow 0 \text { a.s. }
$$

PROOF: Let $x_{n k} \stackrel{2}{\rightarrow} x_{\infty k}$. By Lemma $1,\left\{x_{\infty k}: k \geq 1\right\}$ is an exchangeable sequence of random elements. Thus for $\varepsilon>0$, and by (2.6)

$$
\begin{aligned}
& P\left[\sup _{n \geq m}|| \frac{1}{n} \sum_{k=1}^{n} x_{n k} \|>\varepsilon\right] \leq \underset{n \geq m}{P}\left[\sup _{n=m}|| \frac{1}{n} \sum_{k=1}^{n} x_{n k}-\frac{1}{n} \sum_{k=1}^{n} x_{\infty k}||>\frac{\varepsilon}{2}\right] \\
& +P\left[\sup _{n \geq m}|| \frac{1}{n} \sum_{k=1}^{n} x_{\infty k} \|>\frac{\varepsilon}{2}\right] \\
& =P\left[\sup _{n \geq m}|| E\left(X_{n 1} \mid U_{\infty n}\right)-E\left(X_{\infty 1} \mid U_{\infty n}\right)||>\frac{\varepsilon}{2}\right] \\
& +P\left[\sup _{n \geq m}|| \frac{1}{n} \sum_{k=1}^{n} x_{\infty k}||>\frac{\varepsilon}{2}\right]
\end{aligned}
$$




$$
\begin{aligned}
& =P\left[\sup _{n \geq m}|| E\left(X_{n 1}-X_{\infty 1} \mid U_{\infty n}\right)||>\frac{\varepsilon}{2}\right] \\
& +P\left[\sup _{n \geq m}|| \frac{1}{n} \sum_{k=1}^{n} X_{\infty k}||>\frac{\varepsilon}{2}\right] .
\end{aligned}
$$

Now by $(2.1)$,

$$
P\left[\sup _{n \geq m}|| \frac{1}{n} \sum_{k=1}^{n} x_{\infty k} \|>\frac{\varepsilon}{2}\right]=\int_{F} P_{\nu}\left[\sup _{n \geq m}|| \frac{1}{n} \sum_{k=1}^{n} x_{\infty k} \|>\frac{\varepsilon}{2}\right] d \mu_{\infty}\left(P_{\nu}\right),
$$

where $\left\{x_{\infty k}: k \geq 1\right\}$ are independent, identically distributed random elements with respect to $P_{\nu^{\prime}}$ By Lemma $2, E_{\nu}\left(X_{\infty 1}\right)=0$, with $\mu_{\infty}$-probability one and it follows from Mourier's strong law of large numbers for random elements that for almost each $P_{\nu}$

$$
\mathrm{P}_{\nu}\left[\sup _{\mathrm{n}>\mathrm{m}}|| \frac{1}{\mathrm{n}} \sum_{\mathrm{k}=1}^{\mathrm{n}} \mathrm{x}_{\infty \mathrm{k}}||>\frac{\varepsilon}{2}\right] \rightarrow 0 \text { as } \mathrm{m} \rightarrow \infty .
$$

Hence, by the bounded convergence theorem,

$$
\begin{aligned}
& \left.\underset{n \geq m}{P} \underset{\sup _{n}}{ } \mid \frac{1}{n} \sum_{k=1}^{n} x_{\infty k} \|>\frac{\varepsilon}{2}\right] \\
& =\int_{F} P_{V}\left[\sup _{n \geq m}|| \frac{1}{n} \sum_{k=1}^{n} x_{\infty k} \|>\frac{\varepsilon}{2}\right]
\end{aligned}
$$

goes to 0 as $m \rightarrow \infty$. By (2.5), E( $\left.\left|x_{n 1}-x_{\infty 1} \|\right| u_{n n}\right) \rightarrow 0$ a.s.

Thus,

$$
\begin{aligned}
& P\left[\sup _{n>m}|| E\left(X_{n 1}-x_{\infty 1} \mid U_{\infty n}\right)||>\frac{\varepsilon}{2}\right] \\
& \leq P\left[\sup _{n \geq m} E\left(|| X_{n 1}-x_{\infty 1} \| \mid U_{\infty n}\right)>\frac{\varepsilon}{2}\right] \rightarrow 0 \text { as } m \rightarrow \infty \text {. }
\end{aligned}
$$

Combining (2.7), (2.8) and (2.9), it follows that

$$
P\left[\sup _{n \geq m}|| \frac{1}{n} \sum_{k=1}^{n} X_{n k} \|>\varepsilon\right] \rightarrow 0 \text { as } m \rightarrow \infty,
$$

or that

$$
\left\|\frac{1}{n} \sum_{k=1}^{n} x_{n k}\right\| \rightarrow 0 \text { a.s. }
$$

\section{STRONG LAWS OF LARGE NUMBERS IN TYPE $p$ SPACES}

In this section, strong laws of large numbers for triangular arrays of row-wise exchangeable random elements in type $p+\delta$ separable Banach spaces will be established. Recall that a separable Banach space is said to be of type $p, 1 \leq p \leq 2$, if 
there exists a constant $C$ such that

$$
\left.E|| \Sigma_{k=1}^{n} x_{k}\right|^{p} \leq C \sum_{k=1}^{n} E|| x_{k} \|^{p}
$$

for all independent random elements $x_{1}, \ldots, x_{n}$ with zero means and finite pth moments. Every separable Banach space is type 1. The next result by Woyczynski [7] for sequences of zero mean, independent random elements in E with uniformly bounded tail probabilities is listed for future reference.

THEOREM 2. Let $1<p<2$. The following properties of a Banach space $E$ arc equivalent:

(i) $\mathrm{E}$ is of type $\mathrm{p}+\delta$.

(ii) For any sequence $\left\{x_{i}\right\}$ of zero mean, independent random elements in $E$ with uniformly bounded tail probabilities, the series $\left\|\sum_{n=1}^{\infty} x_{n} / n^{1 / p}\right\|$ converges a.s.

(iii) For any sequence $\left\{x_{i}\right\}$ as in (ii) $\left\|\mathrm{n}^{-1 / \mathrm{p}} \sum_{\mathrm{k}=1}^{\mathrm{n}} \mathrm{x}_{\mathrm{k}}\right\| \rightarrow 0$ a.s.

Since $\left\{x_{\infty k}: k \geq 1\right\}$ are exchangeable, they are identically distributed. Hence, they have uniformly bounded tail probabilities. That is, for all $t \in R$ and $k \geq 1$, $P_{v}\left(|| x_{\infty k}||>t\right) \leq P_{v}\left(|| x_{\infty 1} \|>t\right)$. Thus, a strong convergence result for row-wise exchangeable in type $p$ separable Banach spaces can be obtained using Theorem 2 and the techniques of Theorem 1 .

THEOREM 3. Let $\left\{\mathrm{x}_{\mathrm{nk}}: 1 \leq \mathrm{k} \leq \mathrm{n}, \mathrm{n} \geq 1\right\}$ be an array of random elements in a separable Banach space of type $p+\delta, 1 \leq p<2$. Let $\left\{x_{n k}\right\}$ be exchangeable for each $n$ and let $U_{n n}$ and $U_{o n}$ be the $\sigma$-fields defined in (2.4). If

(i) $\quad|| x_{n 1}-x_{\infty 1}\|\geq\| x_{(n+1), 1}-x_{\infty 1} \|$ for each $n$,

(ii) $E|| x_{n l}-x_{\infty k} \|^{2}=o\left(n^{-2 \alpha}\right)$, where $\alpha=(p-1) / p$, and

(iii) $\rho_{n}(f)=E\left[f\left(x_{n 1}\right) f\left(x_{n 2}\right)\right] \rightarrow 0$ as $n \rightarrow \infty$ for each $f \varepsilon E^{\star}$, then

$$
\left\|1 / \mathrm{n}^{1 / \mathrm{p}} \sum_{\mathrm{k}=1}^{\mathrm{n}} \mathrm{x}_{\mathrm{nk}}\right\| \rightarrow 0 \text { a.s. }
$$

PROOF: Since $E|| x_{n 1}-x_{\infty 1}||^{2}=o\left(n^{-2 \alpha}\right)$, then $x_{n k} \stackrel{2}{\rightarrow} x_{\infty k}$. By Lemma 1 , 
$\left\{x_{\infty k}: k \geq 1\right\}$ is an infinite exchangeable sequence of random elements. Thus, for $\varepsilon>0$, and by (2.6)

$$
\begin{aligned}
& \left.\underset{n=m}{\sup }|| n^{-1 / p} \sum_{k=1}^{n} x_{n k}||>\varepsilon\right] \\
& \leq P\left[\sup _{n>m} \| n^{-1 / P}\left(\sum_{k=1}^{n} x_{n k}-\sum_{k=1}^{n} x_{\infty k}\right)||>\frac{\varepsilon}{2}\right] \\
& +P\left[\sup _{n>m}|| n^{-1 / p} \sum_{k=1}^{n} x_{\infty k}||>\frac{\varepsilon}{2}\right] \\
& =P\left[\sup _{n \geq m}|| n^{\alpha}\left(\frac{1}{n} \sum_{k=1}^{n} x_{n k}-\frac{1}{n} \sum_{k=1}^{n} x_{\infty k}\right)||>\frac{\varepsilon}{2}\right] \\
& +P\left[\sup _{n>m}|| n^{-1 / p} \sum_{k=1}^{n} x_{\infty k}||>\frac{\varepsilon}{2}\right] \\
& =P\left[\sup _{n>m}|| n^{\alpha} E\left(X_{n 1}-x_{\infty 1} \mid U_{\infty n}\right)||>\frac{\varepsilon}{2}\right] \\
& +P\left[\sup _{n \geq m}|| n^{-1 / p} \sum_{k=1}^{n} x_{\infty k} \|>\frac{\varepsilon}{2}\right] .
\end{aligned}
$$

By $(2.1)$

$$
P\left[\sup _{n \geq m}|| n^{-1 / p} \sum_{k=1}^{n} x_{\infty k}||>\frac{\varepsilon}{2}\right]=\int_{F} P_{\nu}\left[\sup _{n \geq m}|| n^{-1 / p} \sum_{k=1}^{n} x_{\infty k}||>\frac{\varepsilon}{2}\right] d \mu_{\infty}\left(P_{\nu}\right),
$$

where $\left\{x_{\infty k}: k \geq 1\right\}$ are independent and identically distributed with respect to $P_{V}$. By Lemma 2, $E_{v}\left(X_{\infty k}\right)=0$. Thus, by Theorem 2 (Theorem 1 for $p=1$ ) ||$n^{-1 / p_{\sum_{k=1}}^{n} X_{\infty k} \| \rightarrow 0}$ a.s. which implies that $P_{v}\left[\sup _{n>m}|| n^{-1 / p} \sum_{k=1}^{n} x_{\infty k} \|>\frac{\varepsilon}{2}\right] \rightarrow 0$ as $m \rightarrow \infty$. By the bounded convergence theorem,

$$
\int_{F} P_{\nu}\left[\sup _{n \geq m}\left\|n^{-1 / p} \sum_{k=1}^{n} x_{\infty k}\right\|>\frac{\varepsilon}{2}\right] d \mu_{\infty}\left(P_{\nu}\right) \rightarrow 0 \text { as } n \rightarrow \infty .
$$

In a manner similar to (2.5), it can be shown that $n^{\alpha}|| E\left(x_{n 1}-x_{\infty 1} \mid U_{\infty n}\right)|| \rightarrow 0$ a.s. This implies that

$$
P\left[\sup _{n \geq m}|| n^{\alpha} E\left(X_{n 1}-X_{\infty 1} \mid U_{\infty n}\right)||>\frac{\varepsilon}{2}\right] \rightarrow 0 \text { as } r_{11} \rightarrow \infty
$$

Hence, it follows that

$$
\left.\underset{n \geq m}{[\sup }|| n^{-1 / p} \sum_{k=1}^{n} x_{n k} \|>\varepsilon\right] \rightarrow 0 \text { as } m \rightarrow \infty,
$$

or that

$$
\left\|\mathrm{n}^{-1 / \mathrm{p}} \sum_{\mathrm{k}=1}^{\mathrm{n}} \mathrm{x}_{\mathrm{nk}}\right\| \rightarrow 0 \text { a.s. }
$$

REMARK. It should be noted that if $\left\|x_{n l}-x_{\infty l}\right\| \rightarrow 0$ a.s., then the condition 
||$x_{n 1}-x_{\infty 1}|| \geq|| x_{(n+1), 1}-x_{\infty 1} \|$ for all $n$ is not needed in the proof of Theorem 3. That is $\left\|x_{n 1}-x_{\infty 1}\right\| \rightarrow 0$ a.s. implies that ||$E\left(x_{n 1}-x_{\infty 1} \mid U_{\infty n}\right)|| \rightarrow 0$ a.s. winich is crucial to the proof of Theorem 3. It is sometimes easier to show directly that ||$E\left(\left(x_{n 1}-x_{\infty n}\right) \mid U_{\infty n}\right)|| \rightarrow 0$ a.s. as will be demonstrated in the kernel density estimation example to follow.

The following example considers the general density estimation problem where $x_{1}, \ldots, x_{n}$ are independent and identically distributed random variables with tne same density function $f$.

EXAMPLE. Let $x_{1}, \ldots, x_{n}$ be independent, identically distributed random variables with common density function $f$. The kernel estimate for $f$ with constant bandwidths $h_{n}$ is given by

$$
f_{n}(t)=\frac{1}{n h_{n}} \sum_{k=1}^{n} K\left(\frac{t-x_{k}}{h_{n}}\right)
$$

where $K$ is often chosen to be a bounded (integrable) kernel with compact support $[a, b]$ and $h_{n} \rightarrow 0$ an $n \rightarrow \infty$. For additional background material, see lelroye and Wagner [8] and Taylor [9]. Let

$$
E=\left\{g \mid g: R \rightarrow R \text { and }|| g||=\left(\int_{-\infty}^{\infty}|g(t)|^{p} d t\right)^{1 / p}<\infty\right\} \text {. }
$$

Thus, E is a separable Banach space of type $\min \{2, p\}$. Define

$$
x_{n k}=\frac{1}{h_{n}}\left(K\left(\frac{t-x_{k}}{h_{n}}\right)-E\left[K\left(\frac{t-x_{1}}{h_{n}}\right)\right]\right)
$$

It is clear that $x_{n k} \in E$ and that $\left\{x_{n k}\right\}$ is independent and hence exchangeable for each $n$. The next proposition will prove directly that ||$E\left(x_{n l} \mid U_{c o n}\right)|| \rightarrow 0$ a.s. In this setting $x_{\infty 1}=0$ a.s.

PROPOSITION 4. Let $x_{n k}=\frac{1}{h}\left(K\left(\frac{t-x_{k}}{h_{n}}\right)-E\left[K\left(\frac{t-X_{1}}{h_{n}}\right)\right]\right)$. Then, $\left\|E\left(X_{n l} \mid U_{\infty n}\right)\right\| \rightarrow 0$ completely (and hence almost surely).

PROOF. Let $U_{n n}=\left\{\sum_{k=1}^{n} x_{n k}, \sum_{k=1}^{n+1} x_{(n+1), k}, \ldots\right\} . U_{n n}=U_{\infty n}$ by (2.4) and since $x_{\infty k}=0$ a.s. for each $k$. For $\varepsilon>0, q \geq 1$, by Markov's inequality, Tonelli's theorem and $(2.6)$

$$
\begin{aligned}
& P\left[|| E\left(X_{n 1} \mid U_{n n}\right)||>\varepsilon\right] \leq \varepsilon^{-2 q} E|| E\left(X_{n 1} \mid U_{n n}\right)||^{2 q} \\
& =\varepsilon^{-2 q_{E}}\left[\left(f_{-\infty}^{\infty}\left|\frac{1}{n h_{n}} \sum_{k=1}^{n}\left(K\left(\frac{t-x_{1}}{h_{n}}\right)-E\left(K\left(\frac{t-x_{1}}{h_{n}}\right)\right)\right)\right|^{p} d t\right)^{1 / p}\right]^{2 q} \\
& \leq \varepsilon^{-2 q} E\left[\int_{-\infty}^{\infty} \mid \frac{1}{n} \sum_{k=1}^{n}\left(\frac{1}{h} K\left(\frac{t-x_{n}}{h_{n}}\right)-\left.E\left(\frac{1}{h_{n}} K\left(\frac{t-x_{1}}{h_{n}}\right)\right)\right|^{2 q} d t\right]\right.
\end{aligned}
$$




$$
\left.\leq \varepsilon^{-2 q} \int_{-\infty}^{\infty} E\left|\frac{1}{n} \sum_{k=1}^{n} \frac{1}{h_{n}} K\left(\frac{t-x_{1}}{h_{n}}\right)-E\left(\frac{1}{h_{n}} K\left(\frac{t-x_{1}}{h_{n}}\right)\right)\right|^{2 q} d t\right]
$$

(Using that $R$ is of type 2 and (2.8) of Taylor [9])

$$
\begin{aligned}
& \leq \varepsilon^{-2 q} \int_{-\infty}^{\infty} n^{q} n^{-2 q} E\left(\left|\frac{1}{h_{n}} K\left(\frac{t-x_{1}}{h_{n}}\right)-E\left(\frac{1}{h_{n}} K\left(\frac{t-x_{1}}{h_{n}}\right)\right)\right|^{2 q}\right) d t \\
& =\varepsilon^{-2 q_{n}-q} E \int_{-\infty}^{\infty} \mid \frac{1}{h} K\left(\frac{t-x_{n}}{h_{n}}\right)-E\left(\left.\frac{1}{h_{n}} K\left(\frac{t-x_{1}}{h_{n}}\right)\right|^{2 q} d t\right. \\
& =\varepsilon^{-2 q_{n}-q} E\left[2(b d d k)^{2 q}(b-a) h_{n}\left(h_{n}\right)^{-2 q}\right] \\
& \leq \frac{C h_{n}}{\left(h_{n}^{2} n\right)} .
\end{aligned}
$$

Letting $h_{n}=o\left(n^{-d}\right), 0 \leq d<\frac{1}{2}$, there exist $q$ such that $\sum_{n=1}^{\infty} h_{n} /\left(h_{n}^{2} n\right)^{q}<\infty$ which implies $\sum_{n=1}^{\infty} P\left[|| E\left(x_{n 1} \mid U_{n n}\right)||>\varepsilon\right]<\infty$. Hence, ||$E\left(x_{n 1} \mid U_{n n}\right)||$ converges completely to 0 .

Depending on the choice of $K,\left\|x_{n 1}\right\|$ may not converge to $0 \equiv x_{\infty 1}$ ( in the example). Also, Theorem 3 and the example emphasizes the importance of $p$ being as large as possible $(<2)$. Moreover, $R, R^{m}$, llilbert spaces and all finite-dimensional Banach spaces are of type 2, and consequently they are type $p+\delta$ for each $p<2$. Finally, it is important to observe that the results are sutstantial new results even for real-valued random variables.

\section{REFERENCES}

1. BLUM, CHERNOFF, ROSENBLATT, AND TEICHER (1958). Central limit theorems for exchangeable process. Canadian J. of Mathematics, 10, 222-229.

2. TAYLOR, R.L. (to appear). Laws of large numbers for dependent random variables. Colloquia Mathematica LIMIT THEOREMS IN PROBABILITY AND STATISTICS.

3. WEBER, N.C. (1980). A martingale approach to central limit theorems for exchangeable random variables. Jour. of Applied Prob. 17, 662-673.

4. BILlingSLEY, P. (1968). Convergence of Probability Measures. Wiley, New York.

5. OLSHEN, R. (1974). A note on exchangeable sequences. Z. Wahrscheinlichkeitstheorie und Verw. Gebiete 28, 317-321.

6. KINGMAN, J.F.C. (1978). Uses of exchangeability. Ann. Prob., 6, 183-197.

7. WOYCZYNSKI, W.A. (1980). On Marcinkiewicz-Zygmund laws of large numbers in Banach spaces and related rates of convergence. Probab. and Math. Statist., 1, 117-131.

8. DeVROYE, L.P. and WAGNER, T.J. (1979). The $\mathrm{L}_{1}$ Convergence of kernel density estimates. Ann. Stat., 7 1136-1139.

9. TAYLOR, R.L. (1982). Convergence of weighted sums of arrays of random elements in type p spaces with application to density estimation. Sankhya A. 44 , 341-351. 


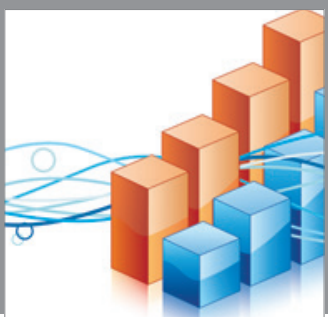

Advances in

Operations Research

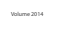

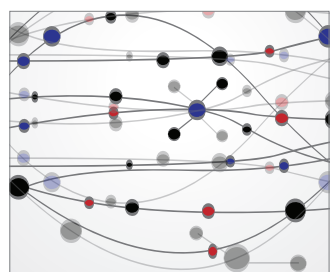

\section{The Scientific} World Journal
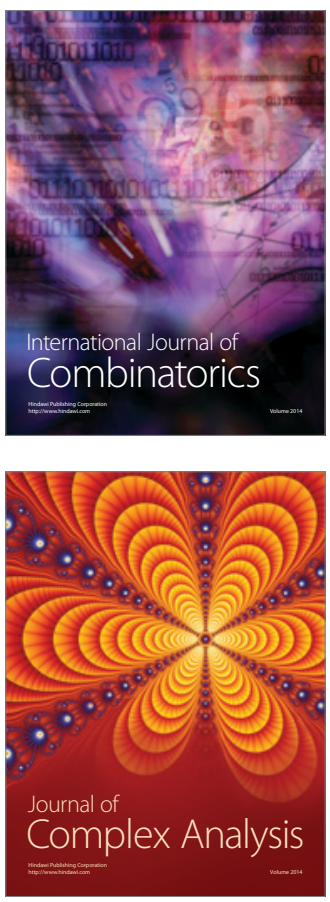

International Journal of

Mathematics and

Mathematical

Sciences
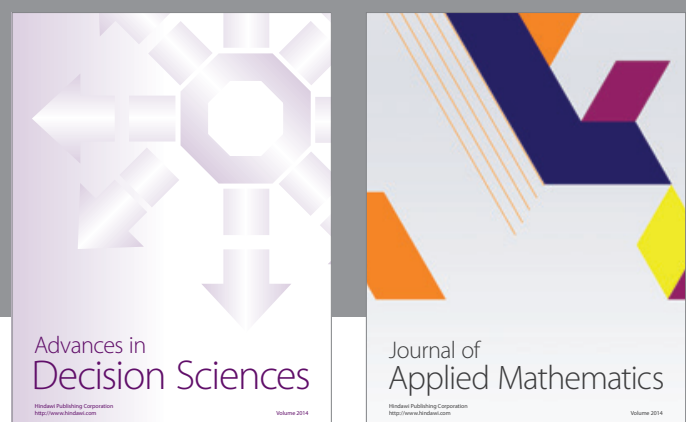

Journal of

Applied Mathematics
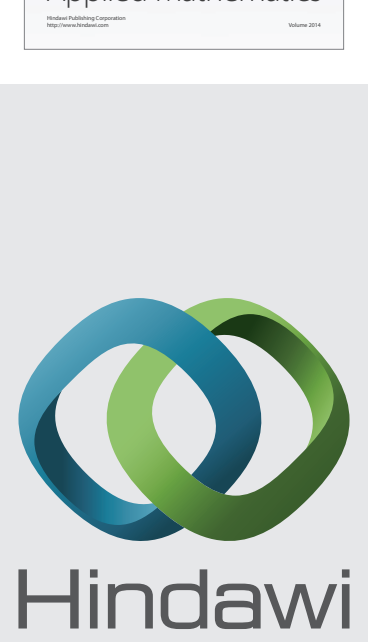

Submit your manuscripts at http://www.hindawi.com
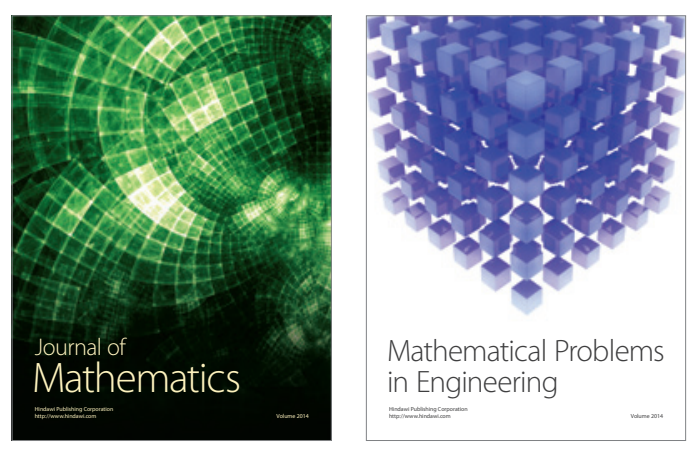

Mathematical Problems in Engineering
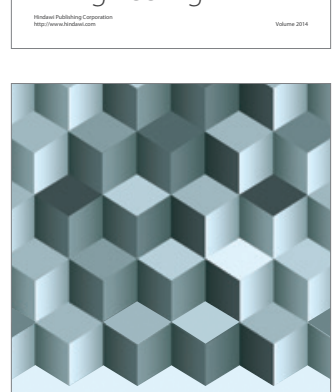

Journal of

Function Spaces
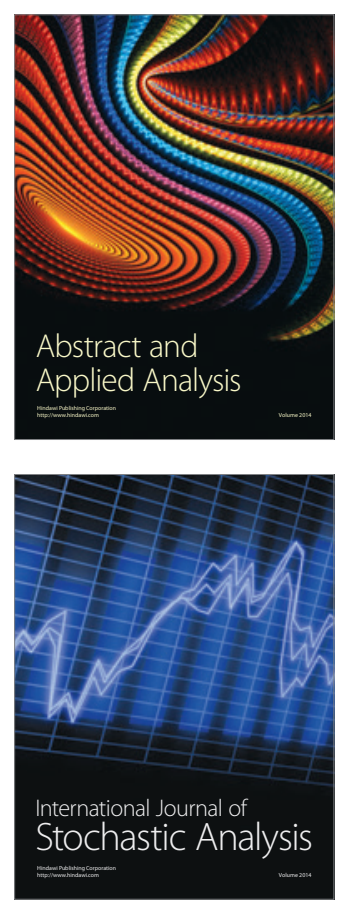

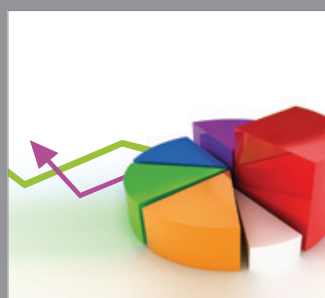

ournal of

Probability and Statistics

Promensencen
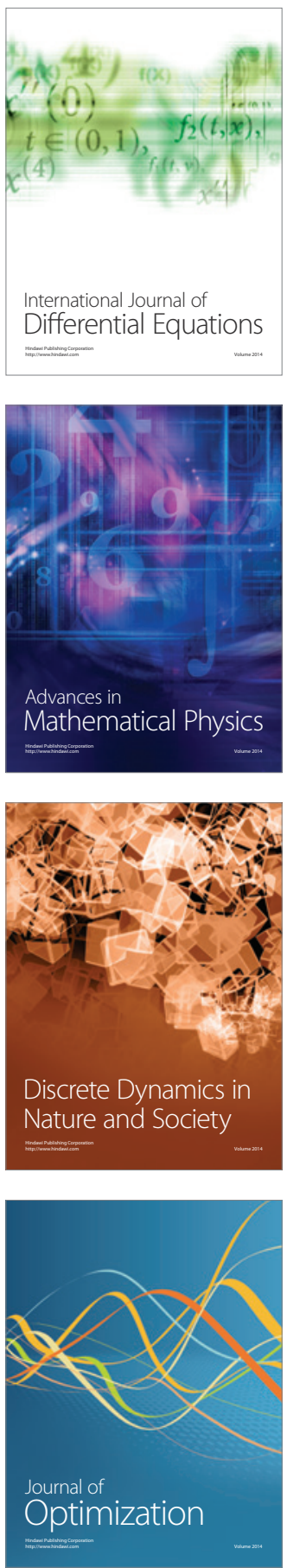